\title{
Let a Hundred Polymers Bloom: Tunable Wetting of Photografted Polymer-Carbon Nitride Surfaces
}

\author{
Paolo Giusto, $*$ Baris Kumru, $*$, Jianrui Zhang, Regina Rothe, and Markus Antonietti
}

Cite This: Chem. Mater. 2020, 32, 7284-7291

Read Online

ABSTRACT: Sunlight is widely used by plants to produce chemical energy fundamental for all life, a process that also serves as an inspiration for science. Recently, light-harvesting semiconductor materials, here, carbon nitrides, have been widely used in photochemistry to catalyze reactions under visible light illumination. We exploited photoactive carbon nitride thin films as a metal-free platform for one-step photografting from polymers on the surface. Here, we observe photoswitchable wetting properties of polystyrene-grafted carbon nitride, which are independent of any classical photoswitchable groups. Dense and thick polymer brush formation with a tunable thickness (up to 700 $\mathrm{nm}$ ) was obtained, allowing for tailor surface properties. Furthermore, exploiting the reciprocal insolubility of water-

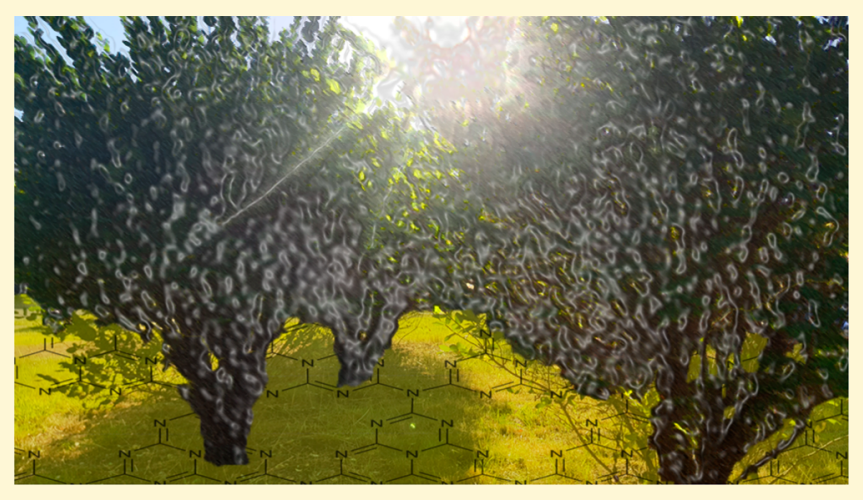
dimethylacrylamide and pentane-styrene solutions, we provide a general method for the synthesis of centimeter-scale carbon nitride-based Janus asymmetric films. It is thought that the simple experimental design based on cheap and common chemicals provides a novel dimension of carbon nitride materials with advanced surface properties.

\section{INTRODUCTION}

The increasing consideration of sustainability and the search for metal-free catalysts led to the rediscovery of polymeric graphitic carbon nitride ( $\mathrm{pCN})$, a metal-free semiconductor absorbing in the visible light range. ${ }^{1,2}$ Such photocatalysts are usually prepared via thermal condensation of abundant, nitrogen-rich precursors, such as urea or melamine, which leads to a polymerized structure with repeating tri-s-triazine units, while photophysical properties of $\mathrm{pCN}$ can be easily tuned via altering precursors or postmodification techniques. $^{3-7}$ As a powder with strong $\pi-\pi$ stacking and slight negative surface charge, $\mathrm{pCN}$ is applied as a heterogeneous photocatalyst in a variety of fields such as $\mathrm{CO}_{2}$ reduction, water splitting, or photoelectrochemistry, only to name a few. ${ }^{7-13}$ Well-defined films of pCN could be particularly interesting for broadening the applications; however, film formation formerly relied on vapor techniques, such as vapor deposition polymerization, thermal vapor condensation and microcontact printing, or spray-coating of organo-modified $\mathrm{pCN}$, both leading in most cases to non-self-supporting and highly defective materials. ${ }^{14-18}$ The formation of well-defined, defect-free $\mathrm{pCN}$ thin films via the chemical vapor deposition method (CVD) was just recently described, for instance with melamine as a precursor. ${ }^{19}$ The thickness-tunable and highly homogeneous CVD method paves the way to employ pCN thin film structures in various applications. Among them, $\mathrm{pCN}$ thin films can be used as a metal-free heterogeneous photoinitiator surface for radical polymerization reactions. ${ }^{20-22}$ The generation of polymer brushes grafted from a flat interface is a powerful approach to tailor the chemical and physical surface properties. ${ }^{23}$ In this context, very recently, Grill and Hecht have launched a quest for new materials and new approaches toward covalent on-surface polymerization that can be met by $\mathrm{pCN}$ thin films. ${ }^{24}$ Covalent on-surface polymerization is of great interest for miniaturized devices for electronics and optoelectronics, but also lab-on-a-chip applications.

The controlled growth of polymer brushes grafted from a substrate is a key step to developing smart surfaces, i.e., surfaces that respond to an external stimulus, such as light. For this purpose, it is necessary to find the right substrate-polymer system. $^{25}$ Stimuli responsive materials modify their surface free energy or morphology, and surface properties like wettability change accordingly. ${ }^{26}$ Various methods have been exploited to control surface wettability, including electric potential, temperature, mechanical stress, $\mathrm{pH}$, and light irradiation. ${ }^{26,27}$ For

Received: April 28, 2020

Revised: July 14, 2020

Published: July 20, 2020 


\section{(a)}

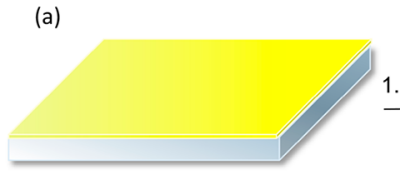

$\stackrel{\text { 1. Add monomer }}{\longrightarrow}$

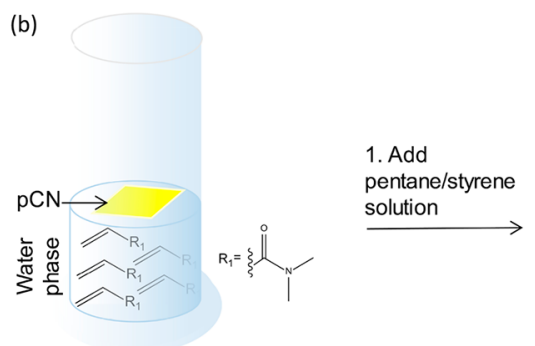

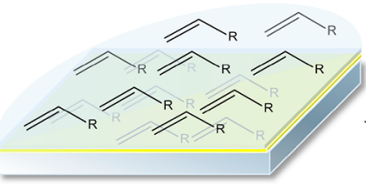

2. Visible light
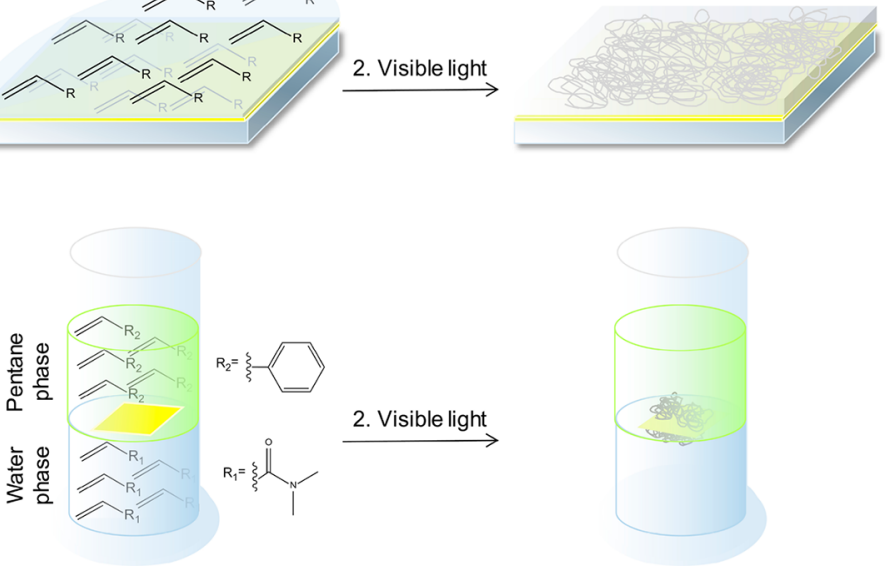

Figure 1. Preparation schemes of light-induced surface grafting of $\mathrm{pCN}$ thin films. (a) Single side pCN-polymer grafted surfaces and (b) 2D-Janus pCN-based superstructures.
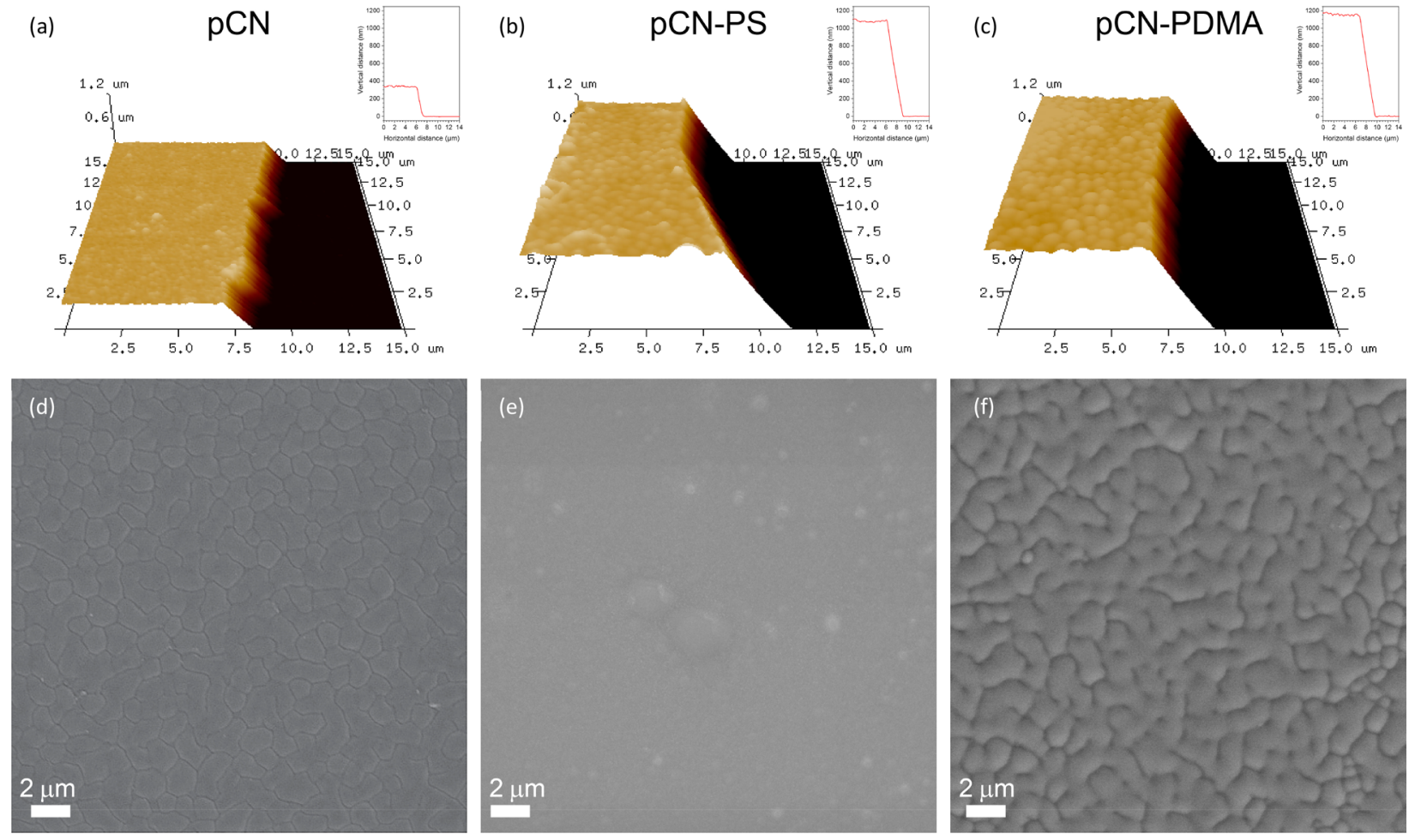

Figure 2. Thickness and surface morphology of $\mathrm{pCN}$ and $\mathrm{pCN}$-polymer grafted structures. (a) AFM images at the film edges of (a) pure pCN film, (b) pCN-PS, and (c) pCN-PDMA after $12 \mathrm{~h}$. The insets highlight the step height measured at the edges of the samples. SEM images of (d) pure pCN film, (e) pCN-PS, and (f) pCN-PDMA.

instance, photoresponsive surfaces that change surface wettability with light find application as antifogging, selfcleaning, and lubricating films, but also in microfluidic optical pumps and valves free of moving parts. ${ }^{26-28}$ Photosensitive semiconductors such as $\mathrm{ZnO}, \mathrm{TiO}_{2}, \mathrm{~V}_{2} \mathrm{O}_{5}, \mathrm{SnO}_{2}$, and other inorganic oxides have shown UV-photoinduced hydrophilicity. ${ }^{29}$ On the other hand, photoswitchable wettability in organic materials relies mostly on the use of photoinduced isomerization of dyes, such as azobenzenes and spiropyranes. $^{26,30,31}$ In these cases, functional polymers are chemically or physically immobilized on the surface of different materials. $^{32}$ Along those lines, Zong and co-workers have recently prepared cotton fabrics dip-coated with a random copolymer containing fluorine and azobenzene functional groups. Upon illumination with UV and Vis light, the cotton fabric shifts from superhydrophilic to superhydrophobic, respectively, due to the UV-triggered isomerization of the azobenzenes groups. ${ }^{30}$ As another example, Wang et al. prepared a patterned surface via atom transfer radical polymerization of a spiropyran monomer on silicon substrate in which, upon illumination with UV and visible light, the contact angle (CA) shifts from $138.8^{\circ}$ to $42.7^{\circ} .{ }^{33}$ However, in all cases, the light-triggered surface properties change requires the presence of photoisomerizable groups on the surface and rather complex chemistry.

Herein, we present a simple and innovative method for onestep photografting of dielectric polymers onto $\mathrm{pCN}$ thin films that allows to control surface properties and shows photo- 
switchable wettability. Eventually, the pCN thin films were used as photoinitiators for radical polymerization reactions, under visible light illumination, for one-step photografting from with common monomers (Figure 1a). This process allows precise control of surface properties such as the contact angle. Moreover, to our surprise, polystyrene films showed photoswitchable wettability under UV illumination without any photoswitchable group, indicating selective charge transport through polystyrene, a property usually not attributed to this most simple mass polymer. Exploiting the simple method to produce covalently polymer-grafted-pCN layers to $\mathrm{pCN}$ films positioned at reciprocally insoluble solvent-solvent interfaces, we have realized amphiphilic pCN-based thin films. In these experiments, we prepared free-standing large area Janus sheets by one-pot grafting of hydrophilic and hydrophobic polymers at the interface of water/pentane monomer solutions (Figure $1 \mathrm{~b}$ ).

\section{RESULTS AND DISCUSSION}

The synthesis of pCN thin films using melamine as a singlesource precursor was recently described. ${ }^{19}$ The as-prepared thin films are highly homogeneous over large areas and are used as substrates for polymer brushes grown using the visible photografting method. Therefore, we chose a set of vinyl monomers, such as styrene and dimethylacrylamide, as commonly available monomers for radical polymerization. The synthetic method requires coating the pCN thin film surface with a vinyl monomer and keeping it under visible illumination for $12 \mathrm{~h}$, while the monomer is re-fed onto the surface every $4 \mathrm{~h}$.

To prove that polymer brushes were grown and covalently grafted from the pCN thin film, the as-prepared polymergrafted pCN samples were immersed in a suitable solvent for the grown polymer brushes, e.g., water for poly(dimethylacrylamide) and tetrahydrofuran for poly(styrene), to remove ungrafted polymers and unreacted monomer residues. Subsequently, the polymer-grafted pCN bilayers were analyzed by Fourier-transform infrared spectroscopy (FT-IR; Figure S1), and the thickness was evaluated by stepheight atomic force microscopy (AFM; Figure 2a-c). The FTIR spectra reveal in all cases the characteristic vibration modes of the respective polymers, different from the bare $\mathrm{pCN}$ thin films, especially in the fingerprint region (Figure S1a). In particular, for the pCN-poly(styrene) (pCN-PS), the spectrum reveals very well-defined peaks at 3034 and $2926 \mathrm{~cm}^{-1}$ attributed to aromatic ring $\mathrm{C}-\mathrm{H}$ and aliphatic $-\mathrm{CH}_{2}-$ stretching modes, respectively, and the out-of-plane aromatic $-\mathrm{C}-\mathrm{H}$ bending at 757 and $698 \mathrm{~cm}^{-1}$, as well as the typical pCN heptazine breathing mode at $810 \mathrm{~cm}^{-1}$ (Figure S1b). ${ }^{19,34}$ The AFM step-height measurements of peeled-off samples deposited on a glass slide reveal that the bare pCN thin film has a thickness of about $330 \mathrm{~nm}$, which increases above 1000 $\mathrm{nm}$ after surface photopolymerization for $\mathrm{pCN}-\mathrm{PS}$ and $\mathrm{pCN}$ PDMA (Figure 2a-c; details of the step height are reported in the insets and in Figure S2). As expected, the grafted polymers do not significantly affect the optical absorption spectrum of pCN thin films, since they are highly transparent above the 350 nm wavelength (Figure S3). The sample surface was further investigated by means of scanning electron microscopy (SEM; Figure $2 \mathrm{~d}-\mathrm{f}$ ) to evaluate the morphology of the bare pCN and the polymer brushes. Analysis of the bare pCN thin film deposited on glass (Figure 1d) shows that the deposition occurs homogeneously and pinhole-free over large surfaces.
Watching closely, the film reveals a microstructure that we attribute to different thermal expansion coefficients of $\mathrm{pCN}$ and glass, causing a different shrinking rate between the film and the substrate during cooling from $550{ }^{\circ} \mathrm{C}$. The surface morphology appears very different after the light-induced grafting of PS (Figure 2e) and PDMA (Figure 2f). With regard to PS-grafted-pCN, the surface looks homogeneously covered and crack-free after the washing step. The small droplet patches present on the surface we attribute to minor impurities of free polystyrene chains being expelled from the grafts (Figure 2e). The PDMA-grafted-pCN surface (Figure $2 \mathrm{f}$ ) is "skin-like" and more rough but nevertheless tight and in the long range homogeneous.

Modifying surfaces of $\mathrm{pCN}$ thin films via grafting with different polymers will modify the surface wettability according to the surface energy of the polymers. For this reason, we prepared surfaces modified with pCN-poly(allylamine) ( $\mathrm{pCN}$ PAA), pCN-poly(methyl methacrylate) (pCN-PMMA), pCNpoly $(N$-isopropylacrylamide) (pCN-PNIPAM), and $\mathrm{pCN}$ poly( $N$-vinylimidazole) (pCN-PVI), in addition to the already mentioned pCN-PS and pCN-PDMA. The surface morphology was determined also for the systems pCN-PAA and pCNPMMA, showing features similar to the previous cases (Figure S4). The bare $\mathrm{pCN}$ thin film has a water contact angle (WCA) of $52.0^{\circ}$, which by modification is tuned in the range of $10.9^{\circ}$ for pCN-PDMA (hydrophilic) up to $132.3^{\circ}$ for pCN-PS (hydrophobic; Figure 3a). This confirms the high flexibility of

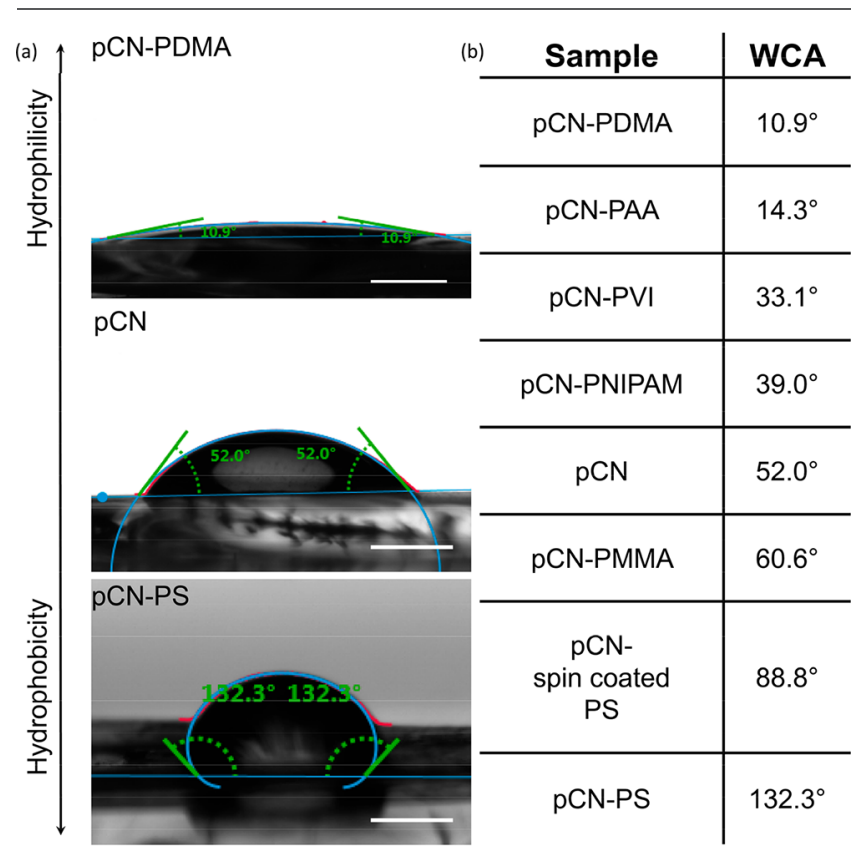

Figure 3. Tunable contact angle of pCN-polymer grafted surfaces. (a) Water contact angles (WCA) of pure pCN film, pCN-PDMA, and pCN-PS; (b) list of the WCA obtained for different pCN-grafted surfaces, ranked from the most wettable to the least wettable. Scale bar $1 \mathrm{~mm}$.

the proposed method, allowing for growing a wide range of polymer brushes from vinylic monomers and by that control the surface properties.

The as-prepared samples' water wettability ranking was determined using the sessile drop method: pCN-PDMA < pCN-PAA $<$ pCN-PVI $<$ pCN-PNIPAM $<$ pCN $<$ pCNPMMA $<$ pCN-PS (listed in Figure $3 b$, WCA images in Figure 

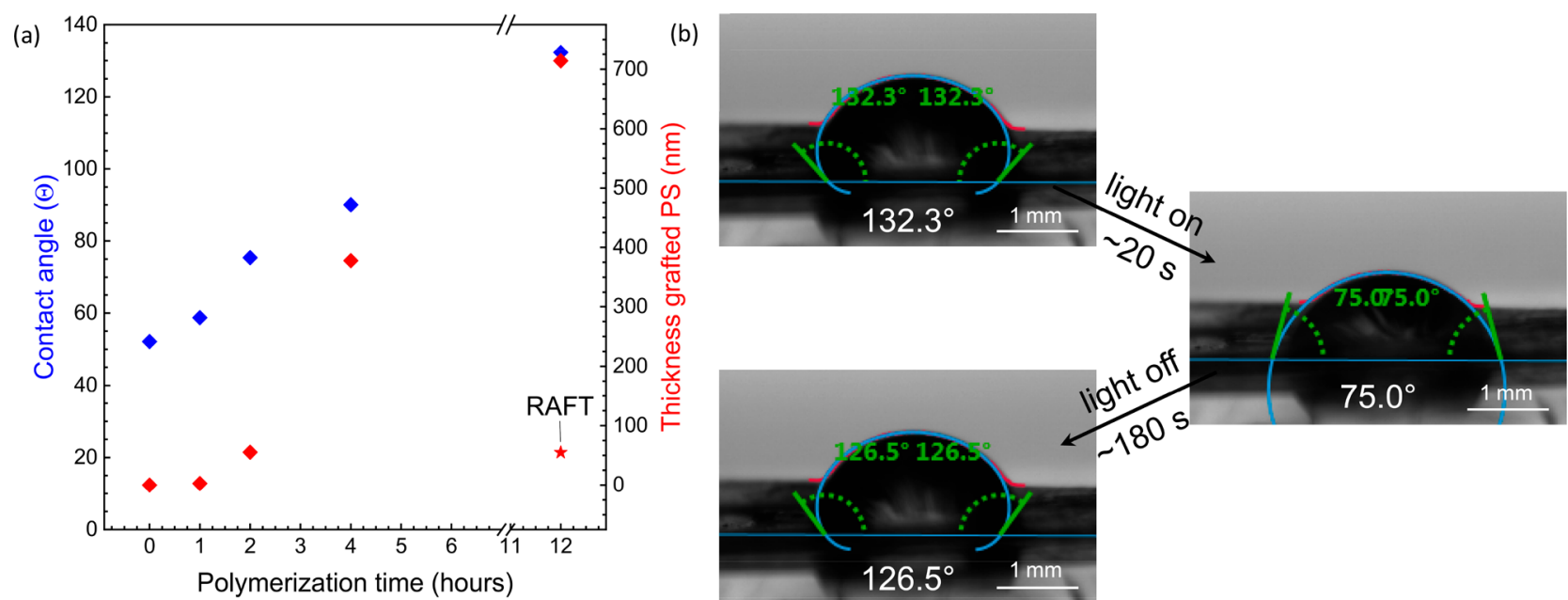

Figure 4. Tunable and photoswitchable contact angle of pCN-PS film. (a) Water contact angles (left axes, in blue) and thickness of the PS-grafted brushes (right axes, in red) of pCN-PS structures for different polymerization times; (b) reversible photoswitchable WCA of pCN-PS under UV illumination with recovery of the initial state in the dark.

S5), in good agreement with surface energy ranking of the bare polymers. It is worth noting that the pCN-PS system shows a very high WCA, much higher with respect to a PS thin film spin-casted on a pCN thin film $\left(88.8^{\circ}\right.$; Figure S6), showing that the as-grown PS brushes possess different properties with respect to commercial PS.

Therefore, we further investigate the PS brush growth on pCN thin film's surface. The visible-light-induced polymerization enhances in this case the thickness by about $700 \mathrm{~nm}$, which corresponds to a stretched chain of about 2800 monomer units, slightly more in a crowded, elongated conformation. From a polymer perspective, such extended growth of polymer brushes by a grafting technique is exceptional. In this case, the amount of PS brushes grafted from the pCN substrate could not be determined by thermal decomposition of pCN-PS in a synthetic air environment (Figure $\mathrm{S} 7 \mathrm{a}-\mathrm{c}$ ). Contrary to the bare $\mathrm{pCN}$ film and a commercial PS, a significant amount of residue remains even at high temperatures for pCN-PS. Despite the tendency of $\mathrm{pCN}$ and PS to thermally decompose at high temperatures, the pCN-PS undergoes a stabilization step, which makes it more thermally resistant than its single counterparts. Similar results were also obtained for pCN-PDMA (Figure S7d). As pointed out in a recent research perspective, the presence of an already formed graphitic carbon, e.g., pCN, could assist in the carbonization of the polymer, and they can lead to materials with a combination of properties that would be difficult to obtain from a single precursor. ${ }^{35}$

Growing polymer brushes on substrates is of high interest to control surface properties in applications such as separation membranes, microfluidic devices, and bioscaffolds, and recently different methods were introduced to control the grafted-layer thickness and the surface properties. ${ }^{36-38}$ The design of stimuli-responsive surfaces is usually achieved by introducing complex functionalities, allowing for obtaining switchable properties with a wider variety of potential applications, such as lab-on-a-chip and self-cleaning surfaces. ${ }^{26,39,40}$ Yet, the density of grafting and chain growth is often low. Usually, polymer brushes grafted on surfaces are in the thickness range of $60-80 \mathrm{~nm}$, although often requiring many synthetic steps. ${ }^{37,41,42}$ In our system, the efficient growth can be attributed to the high photoactivity of $\mathrm{pCN}$ films that act as initiators for the growth of the dielectric polymer: under light illumination the initiating species, i.e., electrons and holes, are formed in the semiconductor and move to surface sites of the pCN. We assume that the first anion and cation radicals very effectively clean the polymerization medium, while the polymerization occurs at rather low radical concentrations and-by geometric constraints of the brush-without the ability to terminate in a simple fashion. The initiating radicals are formed on the surface, and directional growth continues along the final film thickness in a way providing "clean" polymer growth. In order to confirm our assumptions, we have performed the polymerization on the surface in the presence of a RAFT agent (2-cyano-2-propyl benzodithioate), expecting that the resulting chain length is reduced in the presence of competing species. Indeed, AFM confirms only a minor change in surface thickness of the PS film, growing only about $50 \mathrm{~nm}$ (Figure S8), supporting the proposed mechanism. Initiating radicals are taken away from the $\mathrm{pCN}$ surface by transfer agents, such as the RAFT agent, in a similar way to that previously observed also for dispersed bulk pCN, resulting in a smaller PS brush size. ${ }^{20,43,44}$ Therefore, we attribute the radical polymerization governed by holes, as previously reported in our investigations considering carbon nitrides for polymer syntheses. ${ }^{45,46}$

To support a quasi-living character of the radical polymerization on the surface environment, we analyzed the role of polymerization time, i.e., the illumination time of the $\mathrm{pCN}$ film coated with the vinyl monomer, on the PS brush thickness, at 1,2 , and $4 \mathrm{~h}$ of illumination. As expected, the polymer brush thickness increases with the polymerization time, and a similar trend is followed also by the WCA (Figure 4a). After $1 \mathrm{~h}$, the PS-grafted film thickness grows only a few nanometers, causing only a minor change in the pCN contact angle. Between 2 and $4 \mathrm{~h}$, the PS-grafted brush thickness increases dramatically from about 50 to $380 \mathrm{~nm}$, accelerated by the increased local viscosity at higher monomer conversion. The WCA increases accordingly, from 75.4 to $90.0^{\circ}$, thus providing another method to control the surface properties over a wide range, simply by the polymerization time given to the styrene on the pCN surface (Figure S9).

Furthermore, pCN thin films have been used as lightpowered membranes for ion transport. ${ }^{15}$ The ion transport 
mechanism was investigated and attributed to the change in surface charge of $\mathrm{pCN}$, allowing dissolved ions to selectively cross the membrane in the light direction. ${ }^{47}$ Inspired from the light-induced generation of surface charges, we have investigated the effect of UV light illumination on the WCA of the pCN-PS system. As polystyrene is a dielectric material, we expected the charges to "reach through" the PS layer, accounting for its thickness according to the Coulomb law. When exposed to UV illumination, the pCN-PS wettability increases considerably after only $20 \mathrm{~s}$, as shown by the drop of the WCA from $132.3^{\circ}$ to $75.0^{\circ}$; switching off the UV light leads to recovery of the hydrophobic properties in about $3 \mathrm{~min}$ (Figure $4 \mathrm{~b}$ ), and it can be seen even by the naked eye (Video S1). To the best of our knowledge, this is the first time that photoswitchable wettability is shown on PS, which we attribute to the aromatic structure of PS grown directly from the $\mathrm{pCN}$ surface. On the one hand, to our surprise, similar experiments on bare pCN and pCN-PMMA film do show any change in WCA upon light illumination (Videos S2 and S3). On the other hand, the effect of the covalent binding of PS on the $\mathrm{pCN}$ surface was investigated comparing the results of $\mathrm{pCN}-\mathrm{PS}$ with a sample obtained by spin-coating commercial PS on a pCN film surface, and thus without covalent bonds between pCN and PS. As previously mentioned, the spin-coated PS film on pCN WCA increases to $88.8^{\circ}$ (Figure S6), in good agreement with previous results on PS thin films, however, significantly lower with respect to pCN-PS. ${ }^{48}$ The high WCA obtained for pCN-PS obtained by photografting, with respect to the PS spin-coated sample, supports our previous assumption that the grafted polymer brushes grow in a more elongated, stretched conformation. In this regard, Walker and $\mathrm{Li}$ have shown that increasing the polymer radius of gyration increases the hydrophobicity. ${ }^{49}$ Therefore, we can assume that photografted PS brushes on $\mathrm{pCN}$ have a higher gyration radius; i.e., the polymer growth leads to a more elongated conformation, causing higher surface hydrophobicity. Moreover, in this case, no change in WCA was observed upon UV light illumination (Video S4), confirming once more that the change in WCA arises only when covalent bonds between PS and $\mathrm{pCN}$ are formed. All these observations exclude a simple interface charging and action via Coulomb law, further supported by measuring the contact angle change under UV illumination with salty water of pCN-PS: as expected, no change in the contact angle occurs due to the extra ions passivating the interfacial charges (Video S5).

Therefore, our only possible explanation is that covalent grafting of aromatic polymer brushes on the $\mathrm{pCN}$ surface improves charge transfer over the interface, leaving the polystyrene temporarily oxidized, carrying a radical cation on the aromatic rings. This charge transfer builds up slowly $(20 \mathrm{~s})$ and recombines even more slowly (180 s), all time scales which are well above ordinary excited state dynamics. In this model, bare pCN recombines too quickly, while all nonaromatic polymers cannot stabilize the radical cation and are thereby not oxidized. ${ }^{50}$ In other words, the covalent binding of an otherwise insulating plastic layer, such as PS, on our photoactive semiconductor results in directional charge separation and in temporary changes of surface properties with a memory in the minute region.

In the previous part, we exploited the surface photoactivity of pCN films, and we showed different methods to control the surface properties, i.e., by grafting different polymers, polymerization times, and for pCN-PS also reversible light-induced wettability. However, the pCN thin films deposited on glass slides can be peeled from the substrate after soaking in water. Eventually, these films possess two surfaces available for photografting from polymers, allowing for creating asymmetrical superstructures with opposite properties on the two surfaces, e.g., Janus surfaces. To prepare $\mathrm{pCN}$-based large area Janus surfaces, with two different polymer layers, the peeled-off pCN thin film was placed at the interface between two reciprocally insoluble liquids containing a high monomer concentration, e.g., dimethylacrylamide in water (50 vol \%) and styrene in pentane (50 vol \%). Similarly to the previous case, the grafting of polymer brushes onto the $\mathrm{pCN}$ thin film is achieved by illuminating the pCN film at the interface for $24 \mathrm{~h}$ (Figure 1b). The Janus superstructure, constituted of poly(dimethylacrylamide)-pCN-poly(styrene) (PDMA-pCN-PS), was cut and folded to show both surfaces and the pCN edge, the PDMA on top and the PS below, respectively, and analyzed by SEM (Figure 5a,b). The sharp edge of the folded
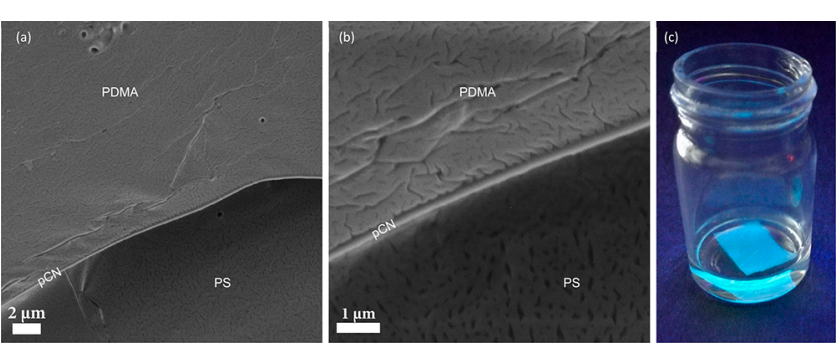

Figure 5. Large area pCN-based Janus surfaces. SEM images of (a) PDMA-pCN-PS large area Janus surface; (b) detail of the pCN-based Janus superstructure (top PDMA; edge pCN; below PS); (c) PDMApCN-PS (PS side up) on water surface under UV illumination.

layer localizes the stiff $\mathrm{pCN}$ film, as highlighted also by energy dispersive X-ray spectroscopy (EDX; Figure S10) where a high intensity signal of the nitrogen element is shown. In the SEM images both sides look very homogeneous but with different morphologies at the two surfaces, and very different from the bare pCN one, with wrinkles, waves, and droplets, typical of polymer layers. This confirms that the method is effective to obtain double surface grafting with different monomers, and therefore the 2D-Janus film over large areas even around 10 $\mathrm{cm}^{2}$ (Figure S11).

Janus $\mathrm{pCN}$ sheets retain their high photoluminescence (Figure 5c) when subjected to UV illumination. We have investigated immersion of Janus sheets in water under UV light. Depending on the hydrophilic or hydrophobic side, Janus pCN sheets fold on the hydrophilic side (PDMA) and open again when contacting water (Video S6). The synthesis of centimeter-sized Janus pCN films, in this facile, one-step synthesis utilizing the interface-based, asymmetric, two-phase grafting polymerization, will be of great interest to develop simple sensors and micromotors with commonly available and cheap precursors. ${ }^{51,52}$

\section{CONCLUSION}

In summary, we presented a generic and simple method to grow different dielectric polymer brushes from vinyl-containing monomers on $\mathrm{pCN}$ thin films, via a one-pot photoinduced polymer-polymer grafting method with visible light. The surface properties of $\mathrm{pCN}$ films can be conveniently tailored by choosing a different monomer and/or with a different polymerization time, over a wide range (from WCA of $10.9^{\circ}$ 
up to $132.3^{\circ}$ ). A very thick and dense polymer brush formation (up to $700 \mathrm{~nm}$ ) was grown directly on the pCN surface, which indicates an efficient surface initiated photopolymerization, poor in radical recombination. Unexpectedly, the polystyrenegrafted $\mathrm{pCN}$ shows also UV light-responsive surface wettability, where UV light illumination grants temporary hydrophilicity, and the initial hydrophobicity is recovered, with switching times in the seconds to minute range. To the best of our knowledge, this is the first time that photoswitchable surface wettability is reported for polystyrene, without photoisomerizable groups obtained by the visible photografting method directly at the $\mathrm{pCN}$ surface. Introducing simple methodologies to engineer surface wettability by UV light will set the base for tailoring surface properties of great interest for future smart devices, which may possess different properties with respect to the single components.

In a second set of experiments, we exploited the $\mathrm{pCN}$ films to generate centimeter scale Janus pCN-based large area surfaces. We believe such components and effects might allow localized solvent transport, smart micromachines, and photocatalytic films responding to different environments.

\section{EXPERIMENTAL SECTION}

Materials. All materials were used as purchased unless noted otherwise: 1-vinyl imidiazole (VI, 99\%, TCI), 2-cyano-2-propyl benzodithioate (97\%, Sigma-Aldrich), allylamine (AA, 99\%, TCI), melamine (99\%, Sigma-Aldrich), methyl methacrylate (MMA, 99\%, Sigma-Aldrich), $\mathrm{N}$-isopropylacrylamide (NIPAM, 97\%, Sigma-Aldrich), N,N-dimethylacrylamide (DMA, 99\%, Sigma-Aldrich), pentane (99\%, Honeywell-Fluka), styrene (99\%, Sigma-Aldrich), poly(styrene) (Polymer Source Inc. $M n=40000, M_{w} / M_{n}=1.05$ ), tetrahydrofuran (THF, 99\%, Sigma-Aldrich), and toluene (99.5\%, Sigma-Aldrich). $50 \mathrm{~W}$ LED chips (365 nm UV light and $420 \mathrm{~nm}$ visible light) were connected to a self-made circuit and cooling system.

Synthesis of $\mathrm{pCN}$ Thin Films. The preparation of $\mathrm{pCN}$ thin films was conducted in a two-zone CVD reactor (planarGROW-3S-OS CVD System from planarTECH), as previously reported elsewhere. ${ }^{19}$ In a typical recipe, $10 \mathrm{~g}$ of melamine was placed in a glass holder and heated up at $300{ }^{\circ} \mathrm{C}\left(10^{\circ} / \mathrm{min}\right)$ in a vacuum (10 Torr). The glass substrates for the deposition were placed in the second zone and heated up at $550{ }^{\circ} \mathrm{C}$ for $90 \mathrm{~min}$. The samples were allowed to cool down naturally at room temperature and collected after breaking the chamber vacuum.

Synthesis of Polymer-Grafted pCN Thin Films. The pCN thin film surfaces were covered with monomers $(0.5 \mathrm{~mL})$ and irradiated via visible light for $12 \mathrm{~h}$ (NIPAM was placed as $50 \mathrm{wt} \%$ aqueous solution). After $12 \mathrm{~h}$, surfaces were washed with water or THF to remove the excess of monomers or unbound polymers, according to their solubility. The films were further dried at room temperature before conducting measurements.

All the polymer grafting was performed via a $50 \mathrm{~W}$ visible light LED source to ensure the following of the experimental conditions that was previously reported in our lab considering $\mathrm{pCN}$ and polymer synthesis. However, it is important to keep in mind that altering the light intensity might affect reaction kinetics and therefore final brush length. ${ }^{53}$

Synthesis of pCN-PS in the Presence of RAFT Agent. The $\mathrm{pCN}$ thin film surface was covered with the styrene monomer $(0.5$ $\mathrm{mL}$ ) including $0.01 \mathrm{~g}$ of 2-cyano-2-propyl benzodithioate as a RAFT agent, and the surface was illuminated via visible light for $12 \mathrm{~h}$. Afterward, the film was immersed in THF for $1 \mathrm{~h}$ to remove the excess of monomers or unbound polymers. The films were further dried at room temperature before conducting measurements.

Time-Dependent Synthesis of pCN-PS. The pCN thin film surface was covered with the styrene monomer $(0.5 \mathrm{~mL})$, and the surface was illuminated via visible light for 1,2 , and $4 \mathrm{~h}$. Afterward, the film was immersed in THF for $1 \mathrm{~h}$ to remove the excess of monomers or unbound polymers. The films were further dried at room temperature before conducting measurements.

Spin-Coated Formation of Noncovalent pCN-PS. Polymer solution was prepared by dissolving PS in toluene $(50 \mathrm{~g} / \mathrm{L})$ in a glass vessel and stirred at room temperature overnight. To prepare the PS thin film, $0.1 \mathrm{~mL}$ of the solution was casted on a pCN thin film substrate and spin-coated at $80 \mathrm{rps}$ for $120 \mathrm{~s}$, until the film was dried.

Synthesis of Janus pCN Thin Films. Water-N,N-dimethylacrylamide $(50 \mathrm{vol} \%)$ and styrene-pentane $(50 \mathrm{vol} \%)$ solutions were previously prepared. Subsequently, the aqueous phase was poured in a vial, and $\mathrm{pCN}$ thin film was placed above the solution. The organic phase was carefully poured above the aqueous phase, and the vial was placed between two visible light LED sources (one from top and one from bottom) irradiating for $12 \mathrm{~h}$ (Figure S12). After reaction, the film was collected with tweezers or a spatula.

Characterization. Fourier transform infrared (FT-IR) spectra were taken on a Nicolet iS 5 FT-IR spectrometer. UV-vis spectra were collected with Shimadzu UV-2600 in the range $800-300 \mathrm{~nm}$ equipped with an integrating sphere. AFM and step-height measurements were obtained using a Dimension 3100 Atomic Force Microscope from Veeco Instruments in the tapping mode and elaborated with NanoScope Analysis 1.9 software. SEM and EDX imaging was performed after $\mathrm{Au} / \mathrm{Pd}$ sputtering of the nonconductive sample in a LEO 1550-Gemini system (acceleration voltage: 2 to 10 $\mathrm{kV}$ ) equipped with an Oxford Instruments X-MAX $80 \mathrm{~mm} 2$ detector. Sessile drop contact angles of water on the coatings were measured at room temperature about $10 \mathrm{~s}$ after placing the drop on the surface with a DSA 10 video contact angle measuring system G10 (Krüss, Germany), and data evaluation was done with DSA version 1.80 .02 software.

\section{ASSOCIATED CONTENT}

\section{Supporting Information}

The Supporting Information is available free of charge at https://pubs.acs.org/doi/10.1021/acs.chemmater.0c01798.

Video S1: Water contact angle shift of pCN-PS under UV illumination (MP4)

Video S2: Water contact angle of bare $\mathrm{pCN}$ under UV illumination (MP4)

Video S3: Water contact angle of pCN-PMMA under UV illumination (MP4)

Video S4: Water contact angle of pCN-spin coated PS under UV illumination (MP4)

Video S5: Contact angle of pCN-PS with salty water under UV illumination (MP4)

Video S6: Janus film bending and folding and opening at contact with water (MP4)

Additional films characterization (FTIR, AFM, UV-vis absorption, SEM and EDX), TGA, WCA, setup for preparation of Janus films (PDF)

\section{AUTHOR INFORMATION}

\section{Corresponding Authors}

Paolo Giusto - Max Planck Institute of Colloids and Interfaces, 14476 Potsdam, Germany; 이이.org/0000-0003-41816500; Email: Paolo.Giusto@mpikg.mpg.de

Baris Kumru - Max Planck Institute of Colloids and Interfaces, 14476 Potsdam, Germany; (1) orcid.org/0000-0002-12034019; Email: Baris.Kumru@mpikg.mpg.de

\section{Authors}

Jianrui Zhang - Max Planck Institute of Colloids and Interfaces, 14476 Potsdam, Germany

Regina Rothe - Max Planck Institute of Colloids and Interfaces, 14476 Potsdam, Germany 
Markus Antonietti - Max Planck Institute of Colloids and Interfaces, 14476 Potsdam, Germany

Complete contact information is available at: https://pubs.acs.org/10.1021/acs.chemmater.0c01798

\section{Author Contributions}

${ }^{\ddagger}$ These authors contributed equally. P.G. and B.K. conceived the idea. P.G. and R.R. prepared the pCN thin films. P.G. and made the absorbance, FTIR, and SEM measurements. B.K. made the light-induced surface polymerization. B.K. and J.Z. made the contact angle measurements. P.G., B.K., and M.A. wrote the manuscript. All authors have given approval to the final version of the manuscript.

Notes

The authors declare no competing financial interest.

\section{ACKNOWLEDGMENTS}

The authors thank the Max Planck Society for funding. We are thankful to Irina Berndt for AFM measurements and the electrical workshop at Max Planck Institute of Colloids and Interfaces for helping with visible light (LED) setups. The authors want to thank Dr. Majd Al-Naji for the support and the useful discussions during the development of this work.

\section{REFERENCES}

(1) Hu, C.; Lin, Y. R.; Yang, H. C. Recent Developments in Graphitic Carbon Nitride Based Hydrogels as Photocatalysts. ChemSusChem 2019, 12 (9), 1794-1806.

(2) Thomas, A.; Fischer, A.; Goettmann, F.; Antonietti, M.; Müller, J.-O.; Schlögl, R.; Carlsson, J. M. Graphitic carbon nitride materials: variation of structure and morphology and their use as metal-free catalysts. J. Mater. Chem. 2008, 18 (41), 4893-4908.

(3) Bu, X.; Li, J.; Yang, S.; Sun, J.; Deng, Y.; Yang, Y.; Wang, G.; Peng, Z.; He, P.; Wang, X.; Ding, G.; Yang, J.; Xie, X. Surface Modification of C3N4 through Oxygen-Plasma Treatment: A Simple Way toward Excellent Hydrophilicity. ACS Appl. Mater. Interfaces 2016, 8 (45), 31419-31425.

(4) Kumru, B.; Molinari, V.; Dunnebacke, R.; Blank, K. G.; Schmidt, B. V. K. J. Extremely Compressible Hydrogel via Incorporation of Modified Graphitic Carbon Nitride. Macromol. Rapid Commun. 2018, 40 (4), 1800712.

(5) Shalom, M.; Inal, S.; Fettkenhauer, C.; Neher, D.; Antonietti, M. Improving carbon nitride photocatalysis by supramolecular preorganization of monomers. J. Am. Chem. Soc. 2013, 135 (19), 7118-21.

(6) Ghosh, I.; Khamrai, J.; Savateev, A.; Shlapakov, N.; Antonietti, M.; König, B. Organic semiconductor photocatalyst can bifunctionalize arenes and heteroarenes. Science 2019, 365 (6451), 360-366.

(7) Cao, S.; Low, J.; Yu, J.; Jaroniec, M. Polymeric photocatalysts based on graphitic carbon nitride. Adv. Mater. 2015, 27 (13), 21502176.

(8) Lin, J.; Pan, Z.; Wang, X. Photochemical Reduction of CO2by Graphitic Carbon Nitride Polymers. ACS Sustainable Chem. Eng. 2014, 2 (3), 353-358.

(9) Han, Q.; Wang, B.; Zhao, Y.; Hu, C.; Qu, L. A Graphitic-C3N4 "Seaweed" Architecture for Enhanced Hydrogen Evolution. Angew. Chem., Int. Ed. 2015, 54 (39), 11433-7.

(10) Wang, X.; Maeda, K.; Thomas, A.; Takanabe, K.; Xin, G.; Carlsson, J. M.; Domen, K.; Antonietti, M. A metal-free polymeric photocatalyst for hydrogen production from water under visible light. Nat. Mater. 2009, 8 (1), 76.

(11) Xu, J.; Herraiz-Cardona, I.; Yang, X.; Gimenez, S.; Antonietti, M.; Shalom, M. The Complex Role of Carbon Nitride as a Sensitizer in Photoelectrochemical Cells. Adv. Opt. Mater. 2015, 3 (8), 10521058.

(12) Kumru, B.; Barrio, J.; Zhang, J.; Antonietti, M.; Shalom, M.; Schmidt, B. V. K. J. Robust Carbon Nitride-Based Thermoset
Coatings for Surface Modification and Photochemistry. ACS Appl. Mater. Interfaces 2019, 11 (9), 9462-9469.

(13) Savateev, A.; Ghosh, I.; König, B.; Antonietti, M. Photoredox catalytic organic transformations using heterogeneous carbon nitrides. Angew. Chem., Int. Ed. 2018, 57 (49), 15936-15947.

(14) Arazoe, H.; Miyajima, D.; Akaike, K.; Araoka, F.; Sato, E.; Hikima, T.; Kawamoto, M.; Aida, T. An autonomous actuator driven by fluctuations in ambient humidity. Nat. Mater. 2016, 15 (10), 1084.

(15) Xiao, K.; Giusto, P.; Wen, L.; Jiang, L.; Antonietti, M. Nanofluidic Ion Transport and Energy Conversion through Ultrathin Free-Standing Polymeric Carbon Nitride Membranes. Angew. Chem., Int. Ed. 2018, 57 (32), 10123-10126.

(16) Liu, J.; Wang, H.; Chen, Z. P.; Moehwald, H.; Fiechter, S.; van de Krol, R.; Wen, L.; Jiang, L.; Antonietti, M. Microcontact-PrintingAssisted Access of Graphitic Carbon Nitride Films with Favorable Textures toward Photoelectrochemical Application. Adv. Mater. 2015, 27 (4), 712-718.

(17) Bian, J.; Li, Q.; Huang, C.; Li, J.; Guo, Y.; Zaw, M.; Zhang, R.Q. Thermal vapor condensation of uniform graphitic carbon nitride films with remarkable photocurrent density for photoelectrochemical applications. Nano Energy 2015, 15, 353-361.

(18) Cruz, D.; Garcia Cerrillo, J.; Kumru, B.; Li, N.; Dario Perea, J.; Schmidt, B. V.; Lauermann, I.; Brabec, C. J.; Antonietti, M. Influence of Thiazole-Modified Carbon Nitride Nanosheets with Feasible Electronic Properties on Inverted Perovskite Solar Cells. J. Am. Chem. Soc. 2019, 141 (31), 12322-12328.

(19) Giusto, P.; Cruz, D.; Heil, T.; Arazoe, H.; Lova, P.; Aida, T.; Comoretto, D.; Patrini, M.; Antonietti, M. Shine Bright Like a Diamond: New Light on an Old Polymeric Semiconductor. Adv. Mater. 2020, 32 (10), 1908140.

(20) Kiskan, B.; Zhang, J.; Wang, X.; Antonietti, M.; Yagci, Y. Mesoporous Graphitic Carbon Nitride as a Heterogeneous Visible Light Photoinitiator for Radical Polymerization. ACS Macro Lett. 2012, 1 (5), 546-549.

(21) Al-Naji, M.; Puertolas, B.; Kumru, B.; Cruz, D.; Baumel, M.; Schmidt, B. V. K. J.; Tarakina, N. V.; Perez-Ramirez, J. Sustainable Continuous Flow Valorization of $\gamma$-Valerolactone with Trioxane to $\alpha$ Methylene- $\gamma$-Valerolactone over Basic Beta Zeolite. ChemSusChem 2019, 12 (12), 2628-2636.

(22) Cao, Q.; Kumru, B.; Antonietti, M.; Schmidt, B. V. Grafting polymers onto carbon nitride via visible-light-induced photofunctionalization. Macromolecules 2019, 52 (13), 4989-4996.

(23) Wang, S.; Zhang, F.; Fan, J.-b. Interfacial Polymerization: From Chemistry to Functional Materials. Angew. Chem., Int. Ed. 2020, DOI: 10.1002/anie.201916473.

(24) Grill, L.; Hecht, S. Covalent on-surface polymerization. Nat. Chem. 2020, 12 (2), 115-130.

(25) Guo, F.; Guo, Z. Inspired smart materials with external stimuli responsive wettability: a review. RSC $A d v$. 2016, 6 (43), 3662336641.

(26) Wang, S.; Liu, K.; Yao, X.; Jiang, L. Bioinspired surfaces with superwettability: new insight on theory, design, and applications. Chem. Rev. 2015, 115 (16), 8230-8293.

(27) Gras, S. L.; Mahmud, T.; Rosengarten, G.; Mitchell, A.; Kalantar-Zadeh, K. Intelligent control of surface hydrophobicity. ChemPhysChem 2007, 8 (14), 2036-2050.

(28) Li, Y.; Wei, Y.; Liao, J.; Hao, Y.; Ning, C.; Jiang, L.; Wang, S. Surface wettability switched cell adhesion and detachment on conducting polymer nanoarray. Adv. Mater. Interfaces 2016, 3 (19), 1600598.

(29) Lim, H. S.; Kwak, D.; Lee, D. Y.; Lee, S. G.; Cho, K. UV-driven reversible switching of a roselike vanadium oxide film between superhydrophobicity and superhydrophilicity. J. Am. Chem. Soc. 2007, 129 (14), 4128-4129.

(30) Zong, C.; Hu, M.; Azhar, U.; Chen, X.; Zhang, Y.; Zhang, S.; $\mathrm{Lu}, \mathrm{C}$. Smart Copolymer-Functionalized Flexible Surfaces with PhotoSwitchable Wettability: From Superhydrophobicity with "Rose Petal" Effect to Superhydrophilicity. ACS Appl. Mater. Interfaces 2019, 11 (28), 25436-25444. 
(31) Jiang, W.; Wang, G.; He, Y.; Wang, X.; An, Y.; Song, Y.; Jiang, L. Photo-switched wettability on an electrostatic self-assembly azobenzene monolayer. Chem. Commun. 2005, No. 28, 3550-3552.

(32) Hozumi, A.; Jiang, L.; Lee, H.; Shimomura, M. StimuliResponsive Dewetting/Wetting Smart Surfaces and Interfaces; Springer, 2018; Vol. 11.

(33) Wang, D.; Jiao, P.; Wang, J.; Zhang, Q.; Feng, L.; Yang, Z. Fast photo-switched wettability and color of surfaces coated with polymer brushes containing spiropyran. J. Appl. Polym. Sci. 2012, 125 (2), $870-875$.

(34) Olmos, D.; Martin, E.; Gonzalez-Benito, J. New molecular-scale information on polystyrene dynamics in PS and PS-BaTiO 3 composites from FTIR spectroscopy. Phys. Chem. Chem. Phys. 2014, 16 (44), 24339-24349.

(35) Gottlieb, E.; Matyjaszewski, K.; Kowalewski, T. Polymer-Based Synthetic Routes to Carbon-Based Metal-Free Catalysts. Adv. Mater. 2019, 31 (13), 1804626.

(36) Matyjaszewski, K.; Miller, P. J.; Shukla, N.; Immaraporn, B.; Gelman, A.; Luokala, B. B.; Siclovan, T. M.; Kickelbick, G.; Vallant, T.; Hoffmann, H.; Pakula, T. Polymers at Interfaces: Using Atom Transfer Radical Polymerization in the Controlled Growth of Homopolymers and Block Copolymers from Silicon Surfaces in the Absence of Untethered Sacrificial Initiator. Macromolecules 1999, 32 (26), 8716-8724.

(37) Chen, W.-L.; Cordero, R.; Tran, H.; Ober, C. K. 50th Anniversary Perspective: Polymer Brushes: Novel Surfaces for Future Materials. Macromolecules 2017, 50, 4089-4113.

(38) Deng, J.; Wang, L.; Liu, L.; Yang, W. Developments and new applications of UV-induced surface graft polymerizations. Prog. Polym. Sci. 2009, 34 (2), 156-193.

(39) Liu, F.; Urban, M. W. Recent advances and challenges in designing stimuli-responsive polymers. Prog. Polym. Sci. 2010, 35 (12), 3-23.

(40) Ichimura, K.; Oh, S.-K.; Nakagawa, M. Light-Driven Motion of Liquids on a Photoresponsive Surface. Science 2000, 288 (5471), $1624-1626$

(41) Giussi, J. M.; Cortez, M. L.; Marmisolle, W. A.; Azzaroni, O. Practical use of polymer brushes in sustainable energy applications: interfacial nanoarchitectonics for high-efficiency devices. Chem. Soc. Rev. 2019, 48, 814-849.

(42) Ghaleh, H.; Jalili, K.; Maher, B. M.; Rahbarghazi, R.; Mehrjoo, M.; Bonakdar, S.; Abbasi, F. Biomimetic antifouling PDMS surface developed via well-defined polymer brushes for cardiovascular applications. Eur. Polym. J. 2018, 106, 305-317.

(43) Kaya, K.; Kiskan, B.; Kumru, B.; Schmidt, B. V. K. J.; Yagci, Y. An oxygen-tolerant visible light induced free radical polymerization using mesoporous graphitic carbon nitride. Eur. Polym. J. 2020, 122, 109410.

(44) Kumru, B.; Mendoza Mesa, J.; Antonietti, M.; Al-Naji, M. Metal-Free Visible-Light-Induced Dithiol-Ene Clicking via Carbon Nitride to Valorize 4-Pentenoic Acid as a Functional Monomer. ACS Sustainable Chem. Eng. 2019, 7 (21), 17574-17579.

(45) Cao, Q.; Kumru, B.; Antonietti, M.; Schmidt, B. V. Graphitic carbon nitride and polymers: a mutual combination for advanced properties. Mater. Horiz. 2020, 7, 762-786.

(46) Cao, Q.; Heil, T.; Kumru, B.; Antonietti, M.; Schmidt, B. V. Visible-light induced emulsion photopolymerization with carbon nitride as a stabilizer and photoinitiator. Polym. Chem. 2019, 10 (39), 5315-5323.

(47) Xiao, K.; Tu, B.; Chen, L.; Heil, T.; Wen, L.; Jiang, L.; Antonietti, M. Photo-Driven Ion Transport for a Photodetector Based on an Asymmetric Carbon Nitride Nanotube Membrane. Angew. Chem., Int. Ed. 2019, 58 (36), 12574-12579.

(48) Li, Y.; Pham, J. Q.; Johnston, K. P.; Green, P. F. Contact angle of water on polystyrene thin films: Effects of $\mathrm{CO} 2$ environment and film thickness. Langmuir 2007, 23 (19), 9785-9793.

(49) Li, I. T.; Walker, G. C. Signature of hydrophobic hydration in a single polymer. Proc. Natl. Acad. Sci. U. S. A. 2011, 108 (40), 1652716532 .
(50) Li, Q.; Liu, J.; Zhang, X.; Tan, S.; Lu, J.; Zhang, Z. Tuning Dielectric and Energy Storage Properties of Polystyrene Based Polymer Dielectric by Manipulating Dipoles and Their Polarizing Behavior. Phys. Chem. Chem. Phys. 2019, 21, 15712-15724.

(51) Zhang, J.; Grzybowski, B. A.; Granick, S. Janus particle synthesis, assembly, and application. Langmuir 2017, 33 (28), 69646977.

(52) Lova, P.; Giusto, P.; Di Stasio, F.; Manfredi, G.; Paterno, G. M.; Cortecchia, D.; Soci, C.; Comoretto, D. All-polymer methylammonium lead iodide perovskite microcavity. Nanoscale 2019, 11 (18), 8978-8983.

(53) Ryan, M. D.; Pearson, R. M.; French, T. A.; Miyake, G. M. Impact of light intensity on control in photoinduced organocatalyzed atom transfer radical polymerization. Macromolecules 2017, 50 (12), $4616-4622$. 